\title{
Erratum to: On the optimal control of the Schlögl-model
}

\section{Rico Buchholz • Harald Engel • Eileen Kammann • Fredi Tröltzsch}

\section{Erratum to: Comput Optim Appl \\ DOI 10.1007/s10589-013-9550-y}

In the paper cited above, both Fig. 4 and Fig. 15 contain two plots. In both figures, the right plot is incorrect. The corrected figures are shown below.

The online version of the original article can be found under doi:10.1007/s10589-013-9550-y.

R. Buchholz

Physikalisches Institut, Universität Bayreuth, 95447 Bayreuth, Germany

e-mail: rico.buchholz@uni-bayreuth.de

H. Engel

Institut für Theoretische Physik, Technische Universität Berlin, 10623 Berlin, Germany

e-mail: Harald.engel@physik.tu-berlin.de

E. Kammann · F. Tröltzsch (凶)

Institut für Mathematik, Technische Universität Berlin, 10623 Berlin, Germany

e-mail: troeltzsch@math.tu-berlin.de

E. Kammann

e-mail: eileen.kammann@googlemail.com 
This is the right version of Fig. 4:
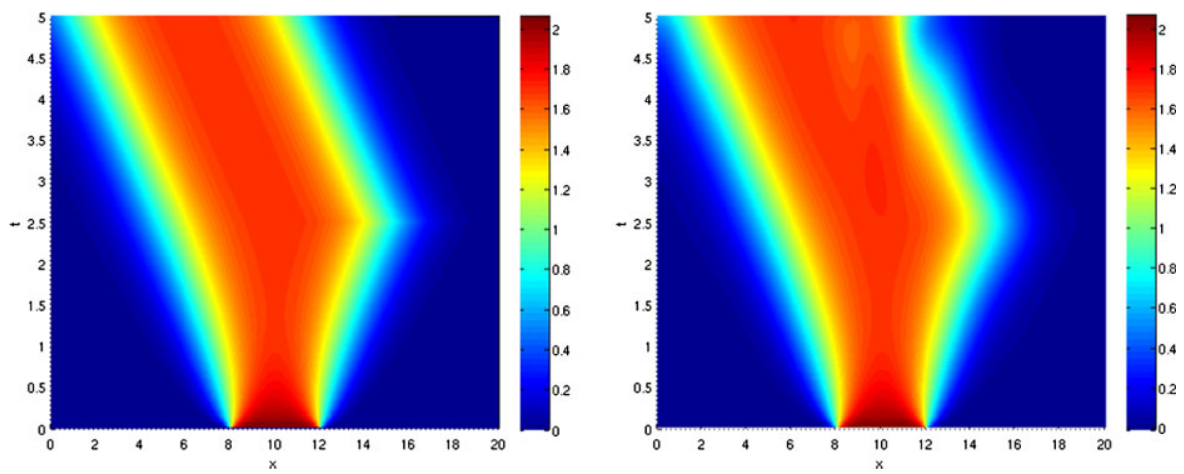

Fig. 4 Acceleration of a wave front, 0.9 sparse control; Desired state (left) and optimal state (right)

Figure 15 should look as follows:
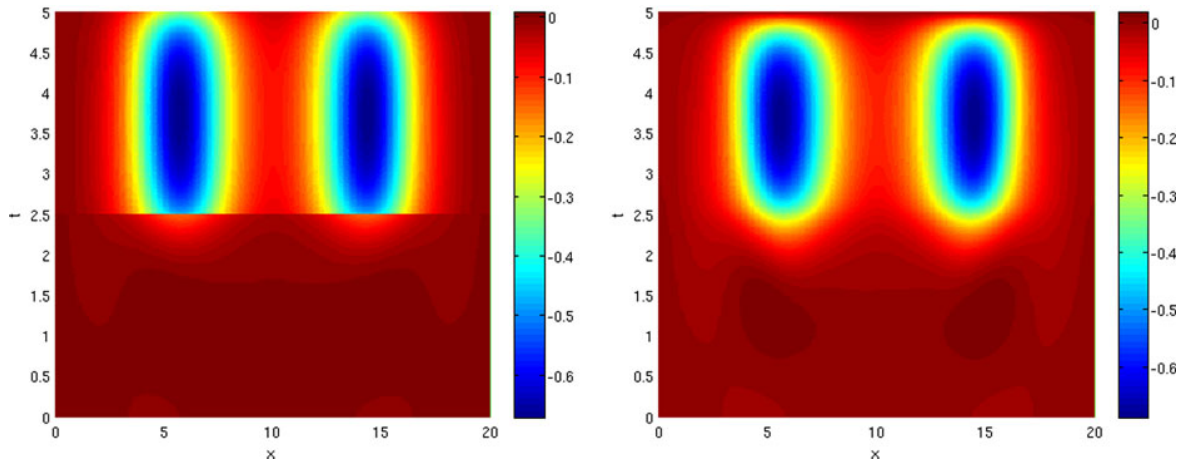

Fig. 15 Optimal controls $\bar{f}$ for Test 3 (left) and Test 4 (right) 